\title{
Malliavin Calculus Applied to Optimal Control of Stochastic Partial Differential Equations with Jumps
}

\author{
Olivier P. Menoukeu', Thilo Meyer-Brandis², Frank Proske², Hassilah \\ B. Salleh²
}

\begin{abstract}
In this paper we employ Malliavin calculus to derive a general stochastic maximum principle for stochastic partial differemtial equations with jumps under partial information. We apply this result to solve an optimal harvesting problem in the presence of partial information. Another application pertains to portfolio optimization under partial observation.
\end{abstract}

AMS Subject Classification: 60G51, 60H40, 60H15

\section{Introduction}

In this paper we aim at using Malliavin calculus to prove a general stochastic maximum principle for stochastic partial differential equations (SPDE's) with jumps under partial information. More precisely, the controlled process is given by a quasilinear stochastic heat equation driven by a Wiener process and a Poisson random measure. Further the control processes are assumed to be adapted to a subfiltration of the filtration generated by the driving noise of the controlled process. Our paper is inspired by ideas developed in Øksendal \& Zhou [14], where the authors establish a general stochastic maximum principle for SDE's based on Malliavin calculus. The results obtained in this paper can be considered a generalization of [14] to the setting of SPDE's.

There is already a vast literature on the stochastic maximum principle. The reader is e.g. referred to [2], [1] , [7], [16], [13], [17] and the references therein. Let us mention that the authors in [2], [16] resort to stochastic maximum principles to study partially observed optimal control problems for diffusions, that is the controls under consideration are based on noisy observations described by the state process. Our paper covers the partial observation case in [2], [16], since we deal with controls being adapted to a general subfiltration of the underlying reference filtration. Further, our Malliavin calculus approach to stochastic control of SPDE's allows for optimization of very general performance functionals. Thus our method is useful to examine control problems of non-Markovian type, which cannot be solved by stochastic dynamic programming. Another important advantage of our technique is that we may relax the assumptions on our Hamiltonian, considerably. For example, we do not need to impose concavity on the Hamiltonian. See e.g. [13], [1]. We remark that the authors in [1] prove a sufficient and necessary maximum principle for partial information control of jump diffusions. However, their method relies on an adjoint equation which often turns out to be unsolvable.

\footnotetext{
${ }^{1}$ Department of Mathematics, University of the Witwatersrand, Private Bag 3, Wits 2050, South Africa

${ }^{2}$ CMA, Department of Mathematics, University of Oslo, P.O. Box 1053 Blindern, N-316 Oslo, Norway. Email: meyerbr@math.uio.no, proske@math.uio.no, hassilas@math.uio.no
} 
We shall give an outline of our paper: In Section 2 we introduce a framework for our partial information control problem. Then in Section 3 we prove a general (sufficient and necessary) maximum principle for SPDE's by invoking Malliavin calculus. See Theorem 3. In Section 4 we use the results of the previous section to solve a partial information optimal harvesting problem (Theorem 5). Further we inquire into an portfolio optimization problem under partial observation. The latter problem boils down to a partial observation problem of jump diffusions, which cannot be captured by the framework of [14].

\section{Framework}

In the following, let $\left\{B_{s}\right\}_{0 \leq s \leq T}$ be a Brownian motion and $\widetilde{N}(d z, d s)=N(d z, d s)-d s \nu(d z)$ a compensated Poisson random measure associated with a Lévy process with Lévy measure $\nu$ on the (complete) filtered probability space $\left(\Omega, \mathcal{F},\left\{\mathcal{F}_{t}\right\}_{0 \leq t \leq T}, P\right)$. In the sequel, we assume that the Lévy measure $\nu$ fulfills

$$
\int_{\mathbb{R}_{0}} z^{2} \nu(d z)<\infty
$$

where $\mathbb{R}_{0}:=\mathbb{R}-\{0\}$.

Consider the controlled stochastic reaction-diffusion equation of the form

$$
\begin{aligned}
d \Gamma(t, x)= & {[L \Gamma(t, x)+b(t, x, \Gamma(t, x), \nabla \Gamma(t, x), u(t, x))] d t } \\
& +\sigma(t, x, \Gamma(t, x), \nabla \Gamma(t, x), u(t, x)) d B_{t} \\
& +\int_{\mathbb{R}} \theta(t, x, \Gamma(t, x), \nabla \Gamma(t, x), u(t, x), z) \widetilde{N}(d z, d t), \\
(t, x) \quad \in \quad & {[0, T] \times G }
\end{aligned}
$$

with boundary condition

$$
\begin{aligned}
\Gamma(0, x) & =\xi(x), x \in \bar{G}, \\
\Gamma(t, x) & =\eta(t, x),(t, x) \in(0, T) \times \partial G .
\end{aligned}
$$

Here $L$ is a partial differential operator of order $m$ and $\nabla$ the gradient acting on the space variable $x \in \mathbb{R}^{n}$ and $G \subset \mathbb{R}^{n}$ is an open set. Further

$$
\begin{array}{rll}
b\left(t, x, \gamma, \gamma^{\prime}, u\right) & :[0, T] \times G \times \mathbb{R} \times \mathbb{R}^{n} \times U \longrightarrow \mathbb{R} \\
\sigma\left(t, x, \gamma, \gamma^{\prime}, u\right) & :[0, T] \times G \times \mathbb{R} \times \mathbb{R}^{n} \times U \longrightarrow \mathbb{R} \\
\theta\left(t, x, \gamma, \gamma^{\prime}, u, z\right) & : & {[0, T] \times G \times \mathbb{R} \times \mathbb{R}^{n} \times U \times \mathbb{R}_{0} \longrightarrow \mathbb{R}} \\
\xi(x) & : & \bar{G} \longrightarrow \mathbb{R} \\
\eta(t, x) & : & (0, T) \times \partial G \longrightarrow \mathbb{R}
\end{array}
$$

are Borel measurable functions, where $U \subset \mathbb{R}$ is a closed convex set. The process

$$
u:[0, T] \times G \times \Omega \longrightarrow U
$$

is called an admissible control if (1) has a unique (strong) solution $\Gamma=\Gamma^{(u)}$ such that $u(t, x)$ is adapted with respect to a subfiltration

$$
\mathcal{E}_{t} \subset \mathcal{F}_{t}, 0 \leq t \leq T,
$$


and such that

$$
\mathbb{E}\left[\int_{0}^{T} \int_{G}|f(t, x, \Gamma(t, x), u(t, x), \omega)| d x d t+\int_{G}|g(x, \Gamma(T, x), \omega)| d x\right]<\infty
$$

for some given $C^{1}$ functions that define the performance functional (see (3) below)

$$
\begin{array}{ll}
f: & :[0, T] \times G \times \mathbb{R} \times U \times \Omega \longrightarrow \mathbb{R}, \\
g & : \quad G \times \mathbb{R} \times \Omega \longrightarrow \mathbb{R} .
\end{array}
$$

A sufficient set of conditions, which ensures the existence of a unique strong solution of (1), is e.g. given by the requirement that the coefficients $b, \sigma, \theta$ satisfy a certain linear growth and Lipschitz condition and that the operator $L$ is bounded and coercive with respect to some Gelfand triple. For more general information on the theory of SPDE's the reader may consult e.g. [4], [9].

Note that one possible subfiltration $\mathcal{E}_{t}$ in $(2)$ is the $\delta$-delayed information given by

$$
\mathcal{E}_{t}=\mathcal{F}_{t-\delta} ; \quad t \geq 0
$$

where $\delta \geq 0$ is a given constant delay.

The $\sigma$-algebra $\mathcal{E}_{t}$ can be interpreted as the entirety of information at time $t$ the controller has access to. We shall denote by $\mathcal{A}=\mathcal{A}_{\mathcal{E}}$ the class of all such admissible controls.

For admissible controls $u \in \mathcal{A}$ define the performance functional

$$
J(u)=\mathbb{E}\left[\int_{0}^{T} \int_{G} f(t, x, \Gamma(t, x), u(t, x), \omega) d x d t+\int_{G} g(x, \Gamma(T, x), \omega) d x\right] .
$$

The optimal control problem is to find the maximum and the maximizer of the performance, i.e. determine the value $J^{*} \in \mathbb{R}$ and the optimal control $u^{*} \in \mathcal{A}$ such that

$$
J^{*}=\sup _{u \in \mathcal{A}} J(u)=J\left(u^{*}\right)
$$

\section{A Generalized Maximum Principle for Stochastic Partial Differential Equations with Jumps}

In this Section we want to derive a general stochastic maximum principle by means of Malliavin calculus. To this end, let us briefly review some basic concepts of this theory. As for definitions and further information on Malliavin calculus see e.g. [12] or [5].

\subsection{Some Elementary Concepts of Malliavin Calculus for Lévy Processes}

Suppose that $B_{t}$ is a Brownian motion on the filtered probability space

$$
\left(\Omega^{(1)}, \mathcal{F}^{(1)},\left\{\mathcal{F}_{t}^{(1)}\right\}_{0 \leq t \leq T}, P^{(1)}\right),
$$


where $\left\{\mathcal{F}_{t}^{(1)}\right\}_{0 \leq t \leq T}$ is the $P^{(1)}$-augmented filtration generated by $B_{t}$ with $\mathcal{F}^{(1)}=\mathcal{F}_{T}^{(1)}$. Analoguously, assume a stochastic basis

$$
\left(\Omega^{(2)}, \mathcal{F}^{(2)},\left\{\mathcal{F}_{t}^{(2)}\right\}_{0 \leq t \leq T}, P^{(2)}\right)
$$

associated with the compensated Poisson random measure $\widetilde{N}(d t, d z)$.

Let us recall the chaos representation property of square integrable functionals of $B_{t}$ and $\widetilde{N}(d t, d z)$ :

(i) If $F \in L^{2}\left(\mathcal{F}^{(1)}, P^{(1)}\right)$ then

$$
F=\sum_{n \geq 0} I_{n}^{(1)}\left(f_{n}\right)
$$

for a unique sequence of symmetric $f_{n} \in L^{2}\left(\lambda^{n}\right)$, where $\lambda$ is the Lebesgue measure and

$$
I_{n}^{(1)}\left(f_{n}\right):=n ! \int_{0}^{T}\left(\int_{0}^{t_{n}} \ldots\left(\int_{0}^{t_{2}} f_{n}\left(t_{1}, \ldots, t_{n}\right) d B_{t_{1}}\right) d B_{t_{2}} \ldots d B_{t_{n}}, n \in \mathbb{N}\right.
$$

the $n$-fold iterated stochastic integral with respect $B_{t}$. Here $I_{n}^{(1)}\left(f_{0}\right):=f_{0}$ for constants $f_{0}$.

(ii) Similarly, if $G \in L^{2}\left(\mathcal{F}^{(2)}, P^{(2)}\right)$, then

$$
G=\sum_{n \geq 0} I_{n}^{(2)}\left(g_{n}\right)
$$

for a unique sequence of kernels $g_{n}$ in $L^{2}\left((\lambda \times \nu)^{n}\right)$, which are symmetric w.r.t. $\left(t_{1}, z_{1}\right), \ldots,\left(t_{n}, z_{n}\right)$. Here $I_{n}^{(2)}\left(g_{n}\right)$ is given by

$I_{n}^{(2)}\left(g_{n}\right):=n ! \int_{0}^{T} \int_{\mathbb{R}_{0}} \int_{0}^{t_{n}} \int_{\mathbb{R}_{0}} \ldots\left(\int_{0}^{t_{2}} \int_{\mathbb{R}_{0}} g_{n}\left(t_{1}, z_{1}, \ldots, t_{n}, z_{n}\right) d B_{t_{1}}\right) \tilde{N}\left(d t_{1}, d z_{1}\right) \ldots \tilde{N}\left(d t_{1}, d z_{1}\right), n \in \mathbb{N}$.

It follows from the Itô isometry that

$$
\|F\|_{L^{2}\left(P^{(1)}\right)}^{2}=\sum_{n \geq 0} n !\left\|f_{n}\right\|_{L^{2}\left(\lambda^{n}\right)}^{2}
$$

and

$$
\|G\|_{L^{2}\left(P^{(2)}\right)}^{2}=\sum_{n \geq 0} n !\left\|g_{n}\right\|_{L^{2}\left((\lambda \times \nu)^{n}\right)}^{2} .
$$

Definition 1 (Malliavin derivatives $D_{t}$ and $D_{t, z}$ ) (i) Denote by $\mathbb{D}_{1,2}^{(1)}$ the stochastic Sobolev space of all $F \in L^{2}\left(\mathcal{F}^{(1)}, P^{(1)}\right)$ with chaos expansion (5) such that

$$
\|F\|_{\mathbb{D}_{1,2}^{(1)}}^{2}:=\sum_{n \geq 0} n n !\left\|f_{n}\right\|_{L^{2}\left(\lambda^{n}\right)}^{2}<\infty .
$$

Then the Malliavin derivative $D_{t}$ of $F \in \mathbb{D}_{1,2}^{(1)}$ in the direction of the Brownian motion $B$ is defined as

$$
D_{t} F=\sum_{n \geq 1} n I_{n-1}^{(1)}\left(\widetilde{f}_{n-1}\right)
$$


where $\widetilde{f}_{n-1}\left(t_{1}, \ldots, t_{n-1}\right):=f_{n}\left(t_{1}, \ldots, t_{n-1}, t\right)$.

(ii) Similarly, let $\mathbb{D}_{1,2}^{(2)}$ be the space of all $G \in L^{2}\left(\mathcal{F}^{(2)}, P^{(2)}\right)$ with chaos representation (6) satisfying

$$
\|G\|_{\mathbb{D}_{1,2}^{(2)}}^{2}:=\sum_{n \geq 0} n n !\left\|g_{n}\right\|_{L^{2}\left((\lambda \times \nu)^{n}\right)}^{2}<\infty .
$$

Then the Malliavin derivative $D_{t, z}$ of $G \in \mathbb{D}_{1,2}^{(2)}$ in the direction of the pure jump Lévy process $\eta_{t}:=\int_{0}^{T} \int_{\mathbb{R}_{0}} z \tilde{N}(d t, d z)$ is defined as

$$
D_{t, z} G:=\sum_{n \geq 1} n I_{n-1}^{(2)}\left(\widetilde{g}_{n-1}\right)
$$

where $\widetilde{g}_{n-1}\left(t_{1}, z_{1}, \ldots, t_{n-1}, z_{n-1}\right):=g_{n}\left(t_{1}, z_{1}, \ldots, t_{n-1}, z_{n-1}, t, z\right)$.

A crucial argument in the proof of our general maximum principle (Theorem 3) rests on duality formulas for the Malliavin derivatives $D_{t}$ and $D_{t, z}[12],[6]$ :

Lemma 2 (Duality formula for $D_{t}$ and $D_{t, z}$ ) (i) Require that $\varphi(t)$ is $\mathcal{F}_{t}^{(1)}$-adapted with $E_{P^{(1)}}\left[\int_{0}^{T} \varphi^{2}(t) d t\right]<\infty$ and $F \in \mathbb{D}_{1,2}^{(1)}$. Then

$$
E_{P^{(1)}}\left[F \int_{0}^{T} \varphi(t) d B_{t}\right]=E_{P^{(1)}}\left[\int_{0}^{T} \varphi(t) D_{t} F d t\right] .
$$

(ii) Assume that $\psi(t, z)$ is $\mathcal{F}_{t}^{(2)}$-adapted with $E_{P^{(2)}}\left[\int_{0}^{T} \int_{\mathbb{R}_{0}} \psi^{2}(t, z) \nu(d z) d t\right]<\infty$ and $G \in$ $\mathbb{D}_{1,2}^{(2)}$. Then

$$
E_{P^{(2)}}\left[G \int_{0}^{T} \int_{\mathbb{R}_{0}} \psi(t, z) \widetilde{N}(d t, d z)\right]=E_{P^{(2)}}\left[\int_{0}^{T} \int_{\mathbb{R}_{0}} \psi(t, z) D_{t, z} G \nu(d z) d t\right] .
$$

In the following we shall confine ourselves to the stochastic basis

$$
\left(\Omega, \mathcal{F},\left\{\mathcal{F}_{t}\right\}_{0 \leq t \leq T}, P\right)
$$

where $\Omega=\Omega^{(1)} \times \Omega^{(2)}, \mathcal{F}=\mathcal{F}^{(1)} \times \mathcal{F}^{(2)}, \mathcal{F}_{t}=\mathcal{F}_{t}^{(1)} \times \mathcal{F}_{t}^{(2)}, P=P^{(1)} \times P^{(2)}$.

We remark that we may state the duality relations in Lemma 2 in terms of $P$.

\subsection{The maximum principle}

In view of the optimization problem (4) we require the following conditions (i)-(v):

(i) The functions $b\left(t, x, \Gamma, \Gamma^{\prime}, u\right), \sigma\left(t, x, \Gamma, \Gamma^{\prime}, u\right), \theta\left(t, x, \Gamma, \Gamma^{\prime}, u, z\right), f(t, x, \Gamma, u, \omega)$, and $g(x, \Gamma, \omega)$ are contained in $C^{1}$ with respect to the arguments $\gamma \in \mathbb{R}$ and $u \in U$. 
(ii) For all $0<t<T$ and all $\mathcal{E}_{t} \otimes \mathcal{B}(\mathbb{R})$ - measurable random variables $\alpha$, the control

$$
\beta_{\alpha}(s, x):=\alpha \cdot \chi_{[t, T]}(s), 0 \leq s \leq T,
$$

where $\chi_{[t, T]}$ denotes the indicator function on $[t, T]$, is an admissible control.

(iii) For all $u, \beta \in \mathcal{A}_{\mathcal{E}}$ with $\beta$ bounded there exists a $\delta>0$ such that

$$
u+y \beta \in \mathcal{A}_{\mathcal{E}}
$$

for all $y \in(-\delta, \delta)$.

(iv) For all $u, \beta \in \mathcal{A}_{\mathcal{E}}$ with $\beta$ bounded the process

$$
Y(t, x)=Y^{\beta}(t, x)=\left.\frac{d}{d y} \Gamma^{(u+y \beta)}(t, x)\right|_{y=0}
$$

exists and

$$
\begin{aligned}
L Y(t, x) & =\left.\frac{d}{d y} L \Gamma^{(u+y \beta)}(t, x)\right|_{y=0} \\
\nabla Y(t, x) & =\left.\frac{d}{d y} \nabla \Gamma^{(u+y \beta)}(t, x)\right|_{y=0}
\end{aligned}
$$

Further suppose that $Y(t, x)$ follows the SPDE

$$
\begin{aligned}
Y(t, x)= & \int_{0}^{t}\left[L Y(s, x)+Y(s, x) \frac{\partial}{\partial \gamma} b(s, x, \Gamma(s, x), \nabla \Gamma(s, x), u(s, x))\right. \\
& \left.+\nabla Y(s, x) \frac{\partial}{\partial \gamma^{\prime}} b(s, x, \Gamma(s, x), \nabla \Gamma(s, x), u(s, x))\right] d s \\
& +\int_{0}^{t}\left[Y(s, x) \frac{\partial}{\partial \gamma} \sigma(s, x, \Gamma(s, x), \nabla \Gamma(t, x), u(s, x))\right. \\
& \left.+\nabla Y(s, x) \frac{\partial}{\partial \gamma^{\prime}} \sigma(s, x, \Gamma(s, x), \nabla \Gamma(t, x), u(s, x))\right] d B_{s} \\
& +\int_{0}^{t} \int_{\mathbb{R}}\left[Y\left(s^{-}, x\right) \frac{\partial}{\partial \gamma} \theta(s, x, \Gamma(s, x), \nabla \Gamma(t, x), u(s, x), z)\right. \\
& \left.+\nabla Y\left(s^{-}, x\right) \frac{\partial}{\partial \gamma^{\prime}} \theta(s, x, \Gamma(s, x), \nabla \Gamma(t, x), u(s, x), z)\right] \widetilde{N}(d z, d s) \\
& +\int_{0}^{t}\left[\beta(s, x) \frac{\partial}{\partial u} b(s, x, \Gamma(s, x), \nabla \Gamma(s, x), u(s, x))\right] d s \\
& +\int_{0}^{t} \beta(s, x) \frac{\partial}{\partial u} \sigma(s, x, \Gamma(s, x), \nabla \Gamma(t, x), u(s, x)) d B_{s} \\
& +\int_{0}^{t} \int_{\mathbb{R}} \beta\left(s^{-}, x\right) \frac{\partial}{\partial u} \theta(s, x, \Gamma(s, x), \nabla \Gamma(t, x), u(s, x), z) \widetilde{N}(d z, d s), \\
\in & {[0, T] \times G, }
\end{aligned}
$$


with

$$
\begin{aligned}
Y(0, x) & =0, x \in \bar{G}, \\
Y(t, x) & =0,(t, x) \in(0, T) \times \partial G .
\end{aligned}
$$

(v) Suppose that for all $u \in \mathcal{A}_{\mathcal{E}}$ the processes

$$
\begin{aligned}
& K(t, x):=\frac{\partial}{\partial \gamma} g(x, \Gamma(T, x), \omega)+\int_{t}^{T} \frac{\partial}{\partial \gamma} f(s, x, \Gamma(s, x), u(s, x), \omega) d s \\
& D_{t} K(t, x):=D_{t} \frac{\partial}{\partial \gamma} g(x, \Gamma(T, x), \omega)+\int_{t}^{T} D_{t}\left(\frac{\partial}{\partial \gamma} f(s, x, \Gamma(s, x), u(s, x), \omega)\right) d s \\
& D_{t, z} K(t, x)=D_{t, z} \frac{\partial}{\partial \gamma} g(x, \Gamma(T, x), \omega)+\int_{t}^{T} D_{t, z}\left(\frac{\partial}{\partial \gamma} f(s, x, \Gamma(s, x), u(s, x), \omega)\right) d s \\
& H_{0}\left(s, x, \gamma, \gamma^{\prime}, u\right):=K(s, x) b\left(s, x, \gamma, \gamma^{\prime}, u\right)+D_{s} K(s, x) \sigma\left(s, x, \gamma, \gamma^{\prime}, u\right) \\
& +\int_{\mathbb{R}} D_{s, z} K(s, x) \theta\left(s, x, \gamma, \gamma^{\prime}, z, u\right) \nu(d z) \\
& p(t, x):=\quad K(t, x)+\int_{t}^{T}\left\{\frac{\partial}{\partial \gamma} H_{0}(s, x, \Gamma(s, x), \nabla \Gamma(s, x), u(s, x))+L^{*} K(s, x)\right. \\
& \left.+\nabla\left(\frac{\partial}{\partial \gamma^{\prime}} H_{0}(s, x, \Gamma(s, x), \nabla \Gamma(s, x), u(s, x))\right)\right\} d s \\
& q(t, x):=\quad D_{t} p(t, x) \\
& r(t, x, z):=\quad D_{t, z} p(t, x) ; t \in[0, T], z \in \mathbb{R}_{0}, x \in G,
\end{aligned}
$$


where $L^{*}$ is the dual operator of $L$, are well-defined and that

$$
\begin{aligned}
& E\left[\int _ { 0 } ^ { T } \int _ { G } \left\{| K ( t , x ) | \left(|L Y(t, x)|+\left|Y(t, x) \frac{\partial}{\partial \gamma} b(t, x, \Gamma(t, x), \nabla \Gamma(t, x), u(t, x))\right|\right.\right.\right. \\
& +\left|\beta(t, x) \frac{\partial}{\partial u} b(t, x, \Gamma(t, x), \nabla \Gamma(t, x), u(t, x))\right| \\
& \left.+\left|\nabla Y(t, x) \frac{\partial}{\partial \gamma^{\prime}} b(t, x, \Gamma(t, x), \nabla \Gamma(t, x), u(t, x))\right|\right) \\
& +\left|D_{t} K(t, x)\right|\left(\left|Y(t, x) \frac{\partial}{\partial \gamma} \sigma(t, x, \Gamma(t, x), \nabla \Gamma(t, x), u(t, x))\right|\right. \\
& +\left|\nabla Y(t, x) \frac{\partial}{\partial \gamma^{\prime}} \sigma(t, x, \Gamma(t, x), \nabla \Gamma(t, x), u(t, x))\right| \\
& \left.+\left|\beta(t, x) \frac{\partial}{\partial u} \sigma(t, x, \Gamma(t, x), \nabla \Gamma(t, x), u(t, x))\right|\right) \\
& +\int_{\mathbb{R}}\left|D_{t, z} K(t, x)\right|\left(\left|Y(t, x) \frac{\partial}{\partial \gamma} \theta(t, x, \Gamma(t, x), \nabla \Gamma(t, x), u(t, x), z)\right|\right. \\
& +\left|\nabla Y(t, x) \frac{\partial}{\partial \gamma^{\prime}} \theta(t, x, \Gamma(t, x), \nabla \Gamma(t, x), u(t, x), z)\right| \\
& \left.+\left|\beta(t, x) \frac{\partial}{\partial u} \theta(t, x, \Gamma(t, x), \nabla \Gamma(t, x), u(t, x), z)\right|\right) \nu(d z) \\
& \left.\left.+\left|\beta(t, x) \frac{\partial}{\partial u} f(t, x, \Gamma(t, x), u(t, x))\right|\right\} d t d x\right] \\
& \infty .
\end{aligned}
$$

Let us comment on that $D_{t} K(t, x)$ and $D_{t, z} K(t, x)$ in (v) exist, if e.g. coefficients $b, \sigma, \theta$ in (1) fulfill a global Lipschitz condition and the operator $L$ is the generator of a strongly continuous semigroup. See e.g. [12], [15] and [3, Section 5].

Now let us introduce the general Hamiltonian

$$
H:[0, T] \times G \times \mathbb{R} \times \mathbb{R}^{n} \times U \times \Omega \longrightarrow \mathbb{R}
$$

by

$$
\begin{aligned}
H\left(t, x, \gamma, \gamma^{\prime}, u, \omega\right):= & f(t, x, \gamma, u, \omega)+p(t, x) b\left(t, x, \gamma, \gamma^{\prime}, u, \omega\right)+D_{t} p(t, x) \sigma\left(t, x, \gamma, \gamma^{\prime}, u, \omega\right) \\
& +\int_{\mathbb{R}} D_{t, z} p(t, x) \theta\left(t, x, \gamma, \gamma^{\prime}, u, z, \omega\right) \nu(d z) .
\end{aligned}
$$

We can now state a general stochastic maximum principle for our partial information control problem (4):

Theorem 3 Retain the conditions (i)-(v). Assume that $\widehat{u} \in \mathcal{A}_{\mathcal{E}}$ is a critical point of the performance functional $J(u)$ in (4), that is

$$
\left.\frac{d}{d y} J(\widehat{u}+y \beta)\right|_{y=0}=0
$$


for all bounded $\beta \in \mathcal{A}_{\mathcal{E}}$. Then

$$
E\left[\int_{G} \frac{\partial}{\partial u} \widehat{H}(t, x, \widehat{\Gamma}(t, x), \nabla \widehat{\Gamma}(t, x), \widehat{u}(t, x)) d x \mid \mathcal{E}_{t}\right]=0 \quad \text { a.e. in }(t, x, \omega)
$$

where

$$
\begin{aligned}
\widehat{\Gamma}(t, x)= & \Gamma^{(\widehat{u})}(t, x), \\
\widehat{H}\left(t, x, \gamma, \gamma^{\prime}, u, \omega\right)= & f(t, x, \gamma, u, \omega)+\widehat{p}(t, x) b\left(t, x, \gamma, \gamma^{\prime}, u, \omega\right)+D_{t} \widehat{p}(t, x) \sigma\left(t, x, \gamma, \gamma^{\prime}, u, \omega\right) \\
& +\int_{\mathbb{R}} D_{t, z} \widehat{p}(t, x) \theta\left(t, x, \gamma, \gamma^{\prime}, u, z, \omega\right) \nu(d z)
\end{aligned}
$$

with

$$
\begin{aligned}
\widehat{p}(t, x)= & \widehat{K}(t, x)+\int_{t}^{T}\left\{\frac{\partial}{\partial \gamma} \widehat{H}_{0}\left(s, x, \widehat{\Gamma}, \widehat{\Gamma}^{\prime}, \widehat{u}, \omega\right)+L^{*} \widehat{K}(s, x)\right. \\
& \left.+\nabla\left(\frac{\partial}{\partial \gamma^{\prime}} H_{0}\left(s, x, \widehat{\Gamma}, \widehat{\Gamma}^{\prime}, \widehat{u}, \omega\right)\right)\right\} d s
\end{aligned}
$$

and

$$
\widehat{K}(t, x)=\frac{\partial}{\partial \gamma} g(x, \widehat{\Gamma}(T, x), \omega)+\int_{t}^{T} \frac{\partial}{\partial \gamma} f(s, x, \widehat{\Gamma}(s, x), \widehat{u}(s, x), \omega) d s .
$$

Remark 4 We remark that in Theorem 3 the patial derivative of $H$ and $H_{0}$ with respect to $u, \gamma$, and $\gamma^{\prime}$ denotes only the differentiation at places where the arguments appear in the coefficients in the definitions (11) and (13).

Proof. Since $\widehat{u} \in \mathcal{A}_{\mathcal{E}}$ is a critical point, there exists for all bounded $\beta \in \mathcal{A}_{\mathcal{E}}$ a $\delta>0$ as in (8). We conclude that

$$
\begin{aligned}
0= & \left.\frac{d}{d y} J(\widehat{u}+y \beta)\right|_{y=0}=E\left[\int _ { 0 } ^ { T } \int _ { G } \left(\frac{\partial}{\partial \gamma} f(s, x, \widehat{\Gamma}(s, x), \widehat{u}(s, x), \omega) \widehat{Y}^{\beta}(s, x)\right.\right. \\
& \left.\left.+\frac{\partial}{\partial u} f(s, x, \widehat{\Gamma}(s, x), \widehat{u}(s, x), \omega) \beta(s, x)\right) d x d s+\int_{G} \frac{\partial}{\partial \gamma} g(x, \widehat{\Gamma}(T, x), \omega) \widehat{Y}^{\beta}(T, x) d x\right],
\end{aligned}
$$


where $\widehat{Y}^{\beta}$ is as defined in (iv) with $u=\widehat{u}$ and fulfills

$$
\begin{aligned}
\widehat{Y}^{\beta}(t, x)= & \int_{0}^{t}\left[L \widehat{Y}^{\beta}(s, x)+\widehat{Y}^{\beta}(s, x) \frac{\partial}{\partial \gamma} b(s, x, \widehat{\Gamma}(s, x), \nabla \widehat{\Gamma}(s, x), \widehat{u}(s, x))\right. \\
& \left.+\nabla \widehat{Y}^{\beta}(s, x) \frac{\partial}{\partial \gamma^{\prime}} b(s, x, \widehat{\Gamma}(s, x), \nabla \widehat{\Gamma}(s, x), \widehat{u}(s, x))\right] d s \\
& +\int_{0}^{t}\left[\widehat{Y}^{\beta}(s, x) \frac{\partial}{\partial \gamma} \sigma(s, x, \widehat{\Gamma}(s, x), \nabla \widehat{\Gamma}(t, x), \widehat{u}(s, x))\right. \\
& \left.+\nabla \widehat{Y}^{\beta}(s, x) \frac{\partial}{\partial \gamma^{\prime}} \sigma(s, x, \widehat{\Gamma}(s, x), \nabla \widehat{\Gamma}(t, x), \widehat{u}(s, x))\right] d B_{s} \\
& +\int_{0}^{t} \int_{\mathbb{R}}\left[\widehat{Y}^{\beta}\left(s^{-}, x\right) \frac{\partial}{\partial \gamma} \theta(s, x, \widehat{\Gamma}(s, x), \nabla \widehat{\Gamma}(t, x), \widehat{u}(s, x), z)\right. \\
& \left.+\nabla \widehat{Y}^{\beta}\left(s^{-}, x\right) \frac{\partial}{\partial \gamma^{\prime}} \theta(s, x, \widehat{\Gamma}(s, x), \nabla \widehat{\Gamma}(t, x), \widehat{u}(s, x), z)\right] \widetilde{N}(d z, d s) \\
& +\int_{0}^{t}\left[\beta(s, x) \frac{\partial}{\partial u} b(s, x, \widehat{\Gamma}(s, x), \nabla \widehat{\Gamma}(s, x), \widehat{u}(s, x))\right] d s \\
& +\int_{0}^{t} \beta(s, x) \frac{\partial}{\partial u} \sigma(s, x, \widehat{\Gamma}(s, x), \nabla \widehat{\Gamma}(t, x), \widehat{u}(s, x)) d B_{s} \\
& +\int_{0}^{t} \int_{\mathbb{R}} \beta\left(s^{-}, x\right) \frac{\partial}{\partial u} \theta(s, x, \widehat{\Gamma}(s, x), \nabla \widehat{\Gamma}(t, x), \widehat{u}(s, x), z) \widetilde{N}(d z, d s) \\
\epsilon & {[0, T] \times G }
\end{aligned}
$$

with

$$
\begin{aligned}
\widehat{Y}^{\beta}(0, x) & =0, x \in \bar{G} \\
\widehat{Y}^{\beta}(t, x) & =0,(t, x) \in(0, T) \times \partial G
\end{aligned}
$$

Thus we obtain that

$$
\begin{aligned}
& E\left[\int_{G} \frac{\partial}{\partial \gamma} g(x, \widehat{\Gamma}(T, x), \omega) \widehat{Y}^{\beta}(T, x) d x\right] \\
= & \int_{G} E\left[\frac{\partial}{\partial \gamma} g(x, \widehat{\Gamma}(T, x), \omega) \widehat{Y}^{\beta}(T, x)\right] d x \\
= & \int_{G} E\left[\frac { \partial } { \partial \gamma } g ( x , \widehat { \Gamma } ( T , x ) , \omega ) \left(\int _ { 0 } ^ { T } \left[L \widehat{Y}^{\beta}(s, x)+\frac{\partial}{\partial \gamma} b(s, x, \widehat{\Gamma}(s, x), \nabla \widehat{\Gamma}(s, x), \widehat{u}(s, x)) \widehat{Y}^{\beta}(s, x)\right.\right.\right. \\
& +\nabla \widehat{Y}^{\beta}(s, x) \frac{\partial}{\partial \gamma^{\prime}} b(s, x, \widehat{\Gamma}(s, x), \nabla \widehat{\Gamma}(s, x), \widehat{u}(s, x)) \\
& \left.+\beta(s, x) \frac{\partial}{\partial u} b(s, x, \widehat{\Gamma}(s, x), \nabla \widehat{\Gamma}(s, x), \widehat{u}(s, x))\right] d s
\end{aligned}
$$




$$
\begin{aligned}
& +\int_{0}^{T}\left[\frac{\partial}{\partial \gamma} \sigma(s, x, \widehat{\Gamma}(s, x), \nabla \widehat{\Gamma}(s, x), \widehat{u}(s, x)) \widehat{Y}^{\beta}(s, x)\right. \\
& +\nabla \widehat{Y}^{\beta}(s, x) \frac{\partial}{\partial \gamma^{\prime}} \sigma(s, x, \widehat{\Gamma}(s, x), \nabla \widehat{\Gamma}(s, x), \widehat{u}(s, x)) \\
& \left.+\frac{\partial}{\partial u} \sigma(s, x, \widehat{\Gamma}(s, x), \nabla \widehat{\Gamma}(s, x), \widehat{u}(s, x)) \beta(s, x)\right] d B_{s} \\
& +\int_{0}^{T} \int_{\mathbb{R}_{0}}\left[\frac{\partial}{\partial \gamma} \theta(s, x, \widehat{\Gamma}(s, x), \nabla \widehat{\Gamma}(s, x), \widehat{u}(s, x), z) \widehat{Y}^{\beta}\left(s^{-}, x\right)\right. \\
& +\nabla \widehat{Y}^{\beta}(s, x) \frac{\partial}{\partial \gamma^{\prime}} \theta(s, x, \widehat{\Gamma}(s, x), \nabla \widehat{\Gamma}(s, x), \widehat{u}(s, x)) \\
& \left.\left.\left.+\frac{\partial}{\partial u} \theta(s, x, \widehat{\Gamma}(s, x), \nabla \widehat{\Gamma}(s, x), \widehat{u}(s, x), z) \beta\left(s^{-}, x\right)\right] \widetilde{N}(d s, d z)\right)\right] d x
\end{aligned}
$$

Then by the duality formulas (Lemma 2) we get that

$$
\begin{aligned}
& E\left[\int_{G} \frac{\partial}{\partial \gamma} g(x, \widehat{\Gamma}(T, x), \omega) \widehat{Y}^{\beta}(T, x) d x\right] \\
= & \int_{G} E\left[\int _ { 0 } ^ { T } \left\{\frac { \partial } { \partial \gamma } g ( x , \widehat { \Gamma } ( T , x ) , \omega ) \left[L \widehat{Y}^{\beta}(s, x)+\frac{\partial}{\partial \gamma} b(s, x, \widehat{\Gamma}(s, x), \nabla \widehat{\Gamma}(s, x), \widehat{u}(s, x)) \widehat{Y}^{\beta}(s, x)\right.\right.\right. \\
& +\frac{\partial}{\partial \gamma^{\prime}} b(s, x, \widehat{\Gamma}(s, x), \nabla \widehat{\Gamma}(s, x), \widehat{u}(s, x)) \nabla Y(s, x) \\
& \left.+\frac{\partial}{\partial u} b(s, x, \widehat{\Gamma}(s, x), \nabla \widehat{\Gamma}(s, x), \widehat{u}(s, x)) \beta(s, x)\right] \\
+ & D_{s}\left(\frac{\partial}{\partial \gamma} g(x, \widehat{\Gamma}(T, x), \omega)\right)\left[\frac{\partial}{\partial \gamma} \sigma(s, x, \widehat{\Gamma}(s, x), \nabla \widehat{\Gamma}(s, x), \widehat{u}(s, x)) \widehat{Y}^{\beta}(s, x)\right. \\
& +\frac{\partial}{\partial \gamma^{\prime}} \sigma(s, x, \widehat{\Gamma}(s, x), \nabla \widehat{\Gamma}(s, x), \widehat{u}(s, x)) \nabla \widehat{Y}^{\beta}(s, x) \\
& \left.+\frac{\partial}{\partial u} \sigma(s, x, \widehat{\Gamma}(s, x), \nabla \widehat{\Gamma}(s, x), \widehat{u}(s, x)) \beta(s, x)\right] \\
& +\int_{\mathbb{R}_{0}}\left\{D _ { s , z } ( \frac { \partial } { \partial \gamma } g ( x , \widehat { \Gamma } ( T , x ) , \omega ) ) \left[\frac{\partial}{\partial \gamma} \theta(s, x, \widehat{\Gamma}(s, x), \nabla \widehat{\Gamma}(s, x), \widehat{u}(s, x), z) \widehat{Y}^{\beta}\left(s^{-}, x\right)\right.\right. \\
& +\frac{\partial}{\partial \gamma^{\prime}} \theta(s, x, \widehat{\Gamma}(s, x), \nabla \widehat{\Gamma}(s, x), \widehat{u}(s, x), z) \nabla \widehat{Y}^{\beta}\left(s^{-}, x\right) \\
& \left.\left.\left.\left.+\frac{\partial}{\partial u} \theta(s, x, \widehat{\Gamma}(s, x), \nabla \widehat{\Gamma}(s, x), \widehat{u}(s, x), z) \beta\left(s^{-}, x\right)\right]\right\} \nu(d z)\right\} d s\right] d x .
\end{aligned}
$$

Further we similarly obtain by duality and Fubini's theorem that 


$$
\begin{aligned}
& E\left[\int_{0}^{T} \int_{G} \frac{\partial}{\partial \gamma} f(s, x, \widehat{\Gamma}(s, x), \widehat{u}(s, x), \omega) \widehat{Y}^{\beta}(s, x) d s d x\right] \\
= & \int_{G} E\left[\int _ { 0 } ^ { T } \left\{\int_{t}^{T} \frac{\partial}{\partial \gamma} f(s, x, \widehat{\Gamma}(s, x), \widehat{u}(s, x), \omega) d s\right.\right. \\
& \cdot\left[L \widehat{Y}^{\beta}(t, x)+\frac{\partial}{\partial \gamma} b(t, x, \widehat{\Gamma}(t, x), \nabla \widehat{\Gamma}(s, x), \widehat{u}(t, x)) \widehat{Y}^{\beta}(t, x)\right. \\
& +\frac{\partial}{\partial u} b(t, x, \widehat{\Gamma}(t, x), \nabla \widehat{\Gamma}(s, x), \widehat{u}(t, x)) \beta(t, x) \\
& \left.+\frac{\partial}{\partial \gamma^{\prime}} b(t, x, \widehat{\Gamma}(t, x), \nabla \widehat{\Gamma}(s, x), \widehat{u}(t, x)) \nabla Y(t, x)\right] d r \\
& +\int_{t}^{T} D_{t}\left(\frac{\partial}{\partial \gamma} f(s, x, \widehat{\Gamma}(s, x), \widehat{u}(s, x), \omega)\right) d s\left[\frac{\partial}{\partial \gamma} \sigma(t, x, \widehat{\Gamma}(t, x), \nabla \widehat{\Gamma}(s, x), \widehat{u}(t, x)) \widehat{Y}^{\beta}(t, x)\right. \\
& +\frac{\partial}{\partial \gamma^{\prime}} \sigma(t, x, \widehat{\Gamma}(t, x), \nabla \widehat{\Gamma}(s, x), \widehat{u}(t, x)) \nabla \widehat{Y}^{\beta}(t, x) \\
& \left.+\frac{\partial}{\partial u} \sigma(t, x, \widehat{\Gamma}(t, x), \nabla \widehat{\Gamma}(s, x), \widehat{u}(t, x)) \beta(t, x)\right] \\
& +\int_{\mathbb{R}_{0}} \int_{t}^{T}\left\{D D_{t, z}\left(\frac{\partial}{\partial \gamma} f(s, x, \widehat{\Gamma}(s, x), \widehat{u}(s, x), \omega)\right) d s\right. \\
& \cdot\left[\frac{\partial}{\partial \gamma} \theta(t, x, \widehat{\Gamma}(t, x), \nabla \widehat{\Gamma}(s, x), \widehat{u}(t, x), z) \widehat{Y}^{\beta}(t, x)\right. \\
& +\frac{\partial}{\partial \gamma^{\prime}} \theta(t, x, \widehat{\Gamma}(t, x), \nabla \widehat{\Gamma}(s, x), \widehat{u}(t, x), z) \nabla \widehat{Y}^{\beta}(t, x) \\
& \left.\left.\left.\left.+\frac{\partial}{\partial u} \theta(t, x, \widehat{\Gamma}(t, x), \nabla \widehat{\Gamma}(s, x), \widehat{u}(t, x), z) \beta(t, x)\right]\right\} \nu(d z)\right\} d t\right] d x
\end{aligned}
$$

Thus by the definition of $\widehat{K}(t, x)$ and notation (iv) it follows that 


$$
\begin{aligned}
& E\left[\int _ { G } \int _ { 0 } ^ { T } \left\{\widehat { K } ( t , x ) \left[L \widehat{Y}^{\beta}(t, x)+\frac{\partial}{\partial \gamma} b(t, x, \widehat{\Gamma}(t, x), \nabla \widehat{\Gamma}(t, x), \widehat{u}(t, x)) \widehat{Y}^{\beta}(t, x)\right.\right.\right. \\
& +\frac{\partial}{\partial \gamma^{\prime}} b(t, x, \widehat{\Gamma}(t, x), \nabla \widehat{\Gamma}(t, x), \widehat{u}(t, x)) \nabla \widehat{Y}^{\beta}(t, x) \\
& \left.+\frac{\partial}{\partial u} b(t, x, \widehat{\Gamma}(t, x), \nabla \widehat{\Gamma}(t, x), \widehat{u}(t, x)) \beta(t, x)\right] \\
& +D_{t} \widehat{K}(t, x)\left[\frac{\partial}{\partial \gamma} \sigma(t, x, \widehat{\Gamma}(t, x), \nabla \widehat{\Gamma}(t, x), \widehat{u}(t, x)) \widehat{Y}^{\beta}(t, x)\right. \\
& +\frac{\partial}{\partial \gamma^{\prime}} \sigma(t, x, \widehat{\Gamma}(t, x), \nabla \widehat{\Gamma}(t, x), \widehat{u}(t, x)) \widehat{Y}^{\beta}(t, x) \\
& \left.+\frac{\partial}{\partial u} \sigma(t, x, \widehat{\Gamma}(t, x), \nabla \widehat{\Gamma}(t, x), \widehat{u}(t, x)) \beta(t, x)\right] \\
& +\int_{\mathbb{R}_{0}}\left\{D _ { t , z } \widehat { K } ( t , x ) \left[\frac{\partial}{\partial \gamma} \theta(r, t, \widehat{\Gamma}(t, x), \nabla \widehat{\Gamma}(t, x), \widehat{u}(t, x), z) \widehat{Y}^{\beta}(r, x)\right.\right. \\
& +\frac{\partial}{\partial \gamma^{\prime}} \theta(r, t, \widehat{\Gamma}(t, x), \nabla \widehat{\Gamma}(t, x), \widehat{u}(t, x), z) \nabla \widehat{Y}^{\beta}(r, x) \\
& \left.\left.+\frac{\partial}{\partial u} \theta(t, x, \widehat{\Gamma}(t, x), \widehat{u}(t, x), z) \beta(t, x)\right]\right\} \nu(d z) \\
& \left.\left.+\frac{\partial}{\partial u} f(t, x, \widehat{\Gamma}(t, x), \widehat{u}(t, x), \omega) \beta(t, x)\right\} d t d x\right] \\
& =0
\end{aligned}
$$

We observe that for all $\beta_{\alpha} \in \mathcal{A}_{\mathcal{E}}$ as defined in (7)

$$
\widehat{Y}^{\beta_{\alpha}}(s, x)=0,0 \leq s \leq t, x \in G .
$$

Then by inspecting (14) we have that

$$
A_{1}+A_{2}+A_{3}+A_{4}=0
$$

where 


$$
\begin{aligned}
A_{1}= & E\left[\int _ { G } \int _ { t } ^ { T } \left\{\widehat{K}(s, x) \frac{\partial}{\partial \gamma} b(s, x) \widehat{Y}^{\beta_{\alpha}}(s, x)+D_{s} \widehat{K}(s, x) \frac{\partial}{\partial \gamma} \sigma(s, x) \widehat{Y}^{\beta_{\alpha}}(s, x)\right.\right. \\
& \left.\left.+\int_{\mathbb{R}} D_{s, z} \widehat{K}(s, x) \frac{\partial}{\partial \gamma} \theta(s, x, z) \widehat{Y}^{\beta_{\alpha}}(s, x) \nu(d z)\right\} d s d x\right] \\
A_{2}= & E\left[\int _ { G } \int _ { t } ^ { T } \left\{\widehat{K}(s, x) \frac{\partial}{\partial u} b(s, x)+D_{s} \widehat{K}(s, x) \frac{\partial}{\partial u} \sigma(s, x)\right.\right. \\
& \left.\left.+\int_{\mathbb{R}} D_{s, z} \widehat{K}(s, x) \frac{\partial}{\partial u} \theta(s, x, z) \nu(d z)+\frac{\partial}{\partial u} f(s, x)\right\} \alpha d s d x\right] \\
A_{3}= & E\left[\int_{G} \int_{t}^{T} \widehat{K}(s, x) L \widehat{Y}^{\beta_{\alpha}}(s, x) d x d t\right] \\
A_{4}= & E\left[\int _ { G } \int _ { t } ^ { T } \left\{\widehat{K}(s, x) \frac{\partial}{\partial \gamma^{\prime}} b(s, x) \nabla \widehat{Y}^{\beta_{\alpha}}(s, x)+D_{s} \widehat{K}(s, x) \frac{\partial}{\partial \gamma^{\prime}} \sigma(s, x) \nabla \widehat{Y}^{\beta_{\alpha}}(s, x)\right.\right. \\
& \left.\left.+\int_{\mathbb{R}} D_{s, z} \widehat{K}(s, x) \frac{\partial}{\partial \gamma^{\prime}} \theta(s, x, z) \nabla \widehat{Y}^{\beta_{\alpha}}(s, x) \nu(d z)\right\} d s d x\right]
\end{aligned}
$$

Note by the definition of $\widehat{Y}^{\beta_{\alpha}}$ that we have

$$
\begin{aligned}
\widehat{Y}^{\beta_{\alpha}}(s, x)= & \int_{t}^{s}\left\{L \widehat{Y}^{\beta_{\alpha}}(r, x)+\widehat{Y}^{\beta_{\alpha}}(r, x) \frac{\partial}{\partial \gamma} b(r, x)+\nabla \widehat{Y}^{\beta_{\alpha}}(r, x) \frac{\partial}{\partial \gamma^{\prime}} b(r, x)+\frac{\partial}{\partial u} b(r, x) \alpha\right\} d r \\
& +\int_{t}^{s}\left\{\widehat{Y}^{\beta_{\alpha}}(r, x) \frac{\partial}{\partial \gamma} \sigma(r, x)+\nabla \widehat{Y}^{\beta_{\alpha}}(r, x) \frac{\partial}{\partial \gamma^{\prime}} \sigma(r, x)+\frac{\partial}{\partial u} \sigma(r, x) \alpha\right\} d B_{r} \\
& +\int_{t}^{s} \int_{\mathbb{R}_{0}}\left\{\widehat{Y}^{\beta_{\alpha}}\left(r^{-}, x\right) \frac{\partial}{\partial \gamma} \theta(r, x, z)+\nabla \widehat{Y}^{\beta_{\alpha}}\left(r^{-}, x\right) \frac{\partial}{\partial \gamma^{\prime}} \theta(r, x, z)\right. \\
& \left.+\frac{\partial}{\partial u} \theta(r, x, z) \alpha\right\} \widetilde{N}(d z, d r) ; \quad 0 \leq t \leq s \leq T
\end{aligned}
$$

Put

$$
\begin{aligned}
\widehat{H}_{0}\left(s, x, \gamma, \gamma^{\prime}, u\right)= & \widehat{K}(s, x) b\left(s, x, \gamma, \gamma^{\prime}, u\right)+D_{s} \widehat{K}(s, x) \sigma\left(s, x, \gamma, \gamma^{\prime}, u\right) \\
& +\int_{\mathbb{R}} D_{s, z} \widehat{K}(s, x) \theta\left(s, x, \gamma, \gamma^{\prime}, z, u\right) \nu(d z)
\end{aligned}
$$

Then

$$
\begin{gathered}
A_{1}=E\left[\int_{G} \int_{t}^{T} \frac{\partial}{\partial \gamma} \widehat{H}_{0}(s, x) \widehat{Y}^{\beta_{\alpha}}(s, x) d s d x\right] \\
=A_{1,1}+A_{1,2}+A_{1,3} \\
A_{2}(t)=E\left[\int_{G} \int_{t}^{T} \frac{\partial}{\partial u}\left(\widehat{H}_{0}(s, x, u)+f(s, x, u)\right) \alpha d s d x\right]
\end{gathered}
$$




$$
\begin{aligned}
A_{3} & =E\left[\int_{G} \int_{t}^{T} \widehat{K}(s, x) L \widehat{Y}^{\beta_{\alpha}}(s, x) d x d t\right] \\
& =E\left[\int_{G} \int_{t}^{T} L^{*} \widehat{K}(s, x) \widehat{Y}^{\beta_{\alpha}}(s, x) d x d t\right] \\
& =A_{3,1}+A_{3,2}+A_{3,3} \\
A_{4}= & E\left[\int_{G} \int_{t}^{T} \nabla\left(\frac{\partial}{\partial \gamma^{\prime}} \widehat{H}_{0}(s, x)\right) \widehat{Y}^{\beta_{\alpha}}(s, x) d s d x\right] \\
= & A_{4,1}+A_{4,2}+4_{1,3}
\end{aligned}
$$

where by duality and Fubini

$$
\begin{aligned}
& A_{1,1}=E\left[\int _ { G } \int _ { t } ^ { T } \left(\int _ { t } ^ { s } \left\{\frac{\partial}{\partial \gamma} \widehat{H}_{0}(s, x) \frac{\partial}{\partial \gamma} b(v, x)+D_{v}\left(\frac{\partial}{\partial \gamma} \widehat{H}_{0}(s, x)\right) \frac{\partial}{\partial \gamma} \sigma(v, x)\right.\right.\right. \\
& \left.+\int_{\mathbb{R}} D_{v, z}\left(\frac{\partial}{\partial \gamma} \widehat{H}_{0}(s, x)\right) \frac{\partial}{\partial \gamma} \theta(v, x, z) \nu(d z)\right\} \widehat{Y}^{\beta_{\alpha}}(v, x) d v \\
& \left.\left.+\int_{t}^{s} \frac{\partial}{\partial \gamma} \widehat{H}_{0}(s, x) L \widehat{Y}^{\beta_{\alpha}}(v, x) d v\right) d s d x\right] \\
& A_{1,2}=E\left[\int _ { G } \int _ { t } ^ { T } \left(\int _ { t } ^ { s } \left\{\frac{\partial}{\partial \gamma} \widehat{H}_{0}(s, x) \frac{\partial}{\partial u} b(v, x)+D_{v}\left(\frac{\partial}{\partial \gamma} \widehat{H}_{0}(s, x)\right) \frac{\partial}{\partial u} \sigma(v, x)\right.\right.\right. \\
& \left.\left.\left.+\int_{\mathbb{R}} D_{v, z}\left(\frac{\partial}{\partial \gamma} \widehat{H}_{0}(s, x)\right) \frac{\partial}{\partial u} \theta(v, x, z) \nu(d z)\right\} d v\right) \alpha d s d x\right] \\
& A_{1,3}=E\left[\int _ { G } \int _ { t } ^ { T } \left(\int _ { t } ^ { s } \left\{\frac{\partial}{\partial \gamma} \widehat{H}_{0}(s, x) \frac{\partial}{\partial \gamma^{\prime}} b(v, x)+D_{v}\left(\frac{\partial}{\partial \gamma} \widehat{H}_{0}(s, x)\right) \frac{\partial}{\partial \gamma^{\prime}} \sigma(v, x)\right.\right.\right. \\
& \left.\left.\left.+\int_{\mathbb{R}} D_{v, z}\left(\frac{\partial}{\partial \gamma} \widehat{H}_{0}(s, x)\right) \frac{\partial}{\partial \gamma^{\prime}} \theta(v, x, z) \nu(d z)\right\} \nabla \widehat{Y}^{\beta_{\alpha}}(v, x) d v\right) d s d x\right] ; \\
& A_{3,1}=E\left[\int _ { G } \int _ { t } ^ { T } \left(\int _ { t } ^ { s } \left\{L^{*} \widehat{K}(s, x) \frac{\partial}{\partial \gamma} b(v, x)+D_{v}\left(L^{*} \widehat{K}(s, x)\right) \frac{\partial}{\partial \gamma} \sigma(v, x)\right.\right.\right. \\
& \left.+\int_{\mathbb{R}_{0}} D_{v, z}\left(L^{*} \widehat{K}(s, x)\right) \frac{\partial}{\partial \gamma} \theta(v, x, z) \nu(d z)\right\} \widehat{Y}^{\beta_{\alpha}}(v, x) d v \\
& \left.\left.+\int_{t}^{s} L^{*} \widehat{K}(s, x) L \widehat{Y}^{\beta_{\alpha}}(v, x) d v\right) d s d x\right] \\
& A_{3,2}=E\left[\int _ { G } \int _ { t } ^ { T } \left(\int _ { t } ^ { s } \left\{L^{*} \widehat{K}(s, x) \frac{\partial}{\partial u} b(v, x)+D_{v}\left(L^{*} \widehat{K}(s, x)\right) \frac{\partial}{\partial u} \sigma(v, x)\right.\right.\right. \\
& \left.\left.\left.+\int_{\mathbb{R}_{0}} D_{v, z}\left(L^{*} \widehat{K}(s, x)\right) \frac{\partial}{\partial u} \theta(v, x, z) \nu(d z)\right\} d v\right) \alpha d s d x\right] ; \\
& A_{3,3}=E\left[\int _ { G } \int _ { t } ^ { T } \left(\int _ { t } ^ { s } \left\{L^{*} \widehat{K}(s, x) \frac{\partial}{\partial \gamma^{\prime}} b(v, x)+D_{v}\left(L^{*} \widehat{K}(s, x)\right) \frac{\partial}{\partial \gamma^{\prime}} \sigma(v, x)\right.\right.\right. \\
& \left.\left.\left.+\int_{\mathbb{R}_{0}} D_{v, z}\left(L^{*} \widehat{K}(s, x)\right) \frac{\partial}{\partial \gamma^{\prime}} \theta(v, x, z) \nu(d z)\right\} \nabla \widehat{Y}^{\beta_{\alpha}}(v, x) d v\right) d s d x\right],
\end{aligned}
$$




$$
\begin{aligned}
A_{4,1}=E & {\left[\int _ { G } \int _ { t } ^ { T } \left(\int _ { t } ^ { s } \left\{\nabla\left(\frac{\partial}{\partial \gamma^{\prime}} \widehat{H}_{0}(s, x)\right) \frac{\partial}{\partial \gamma} b(v, x)+D_{v}\left(\nabla\left(\frac{\partial}{\partial \gamma^{\prime}} \widehat{H}_{0}(s, x)\right)\right) \frac{\partial}{\partial \gamma} \sigma(v, x)\right.\right.\right.} \\
& \left.+\int_{\mathbb{R}_{0}} D_{v, z}\left(\nabla\left(\frac{\partial}{\partial \gamma^{\prime}} \widehat{H}_{0}(s, x)\right)\right) \frac{\partial}{\partial \gamma} \theta(v, x, z) \nu(d z)\right\} \widehat{Y}^{\beta_{\alpha}}(v, x) d v \\
& \left.\left.+\int_{t}^{s} \nabla\left(\frac{\partial}{\partial \gamma^{\prime}} \widehat{H}_{0}(s, x)\right) L \widehat{Y}^{\beta_{\alpha}}(v, x) d v\right) d s d x\right] ; \\
A_{4,2}=E & {\left[\int _ { G } \int _ { t } ^ { T } \left(\int _ { t } ^ { s } \left\{\nabla\left(\frac{\partial}{\partial \gamma^{\prime}} \widehat{H}_{0}(s, x)\right) \frac{\partial}{\partial u} b(v, x)+D_{v}\left(\nabla\left(\frac{\partial}{\partial \gamma^{\prime}} \widehat{H}_{0}(s, x)\right)\right) \frac{\partial}{\partial u} \sigma(v, x)\right.\right.\right.} \\
& \left.\left.\left.+\int_{\mathbb{R}_{\digamma}} D_{v, z}\left(\nabla\left(\frac{\partial}{\partial \gamma^{\prime}} \widehat{H}_{0}(s, x)\right)\right) \frac{\partial}{\partial u} \theta(v, x, z) \nu(d z)\right\} d v\right) \alpha d s d x\right] ; \\
A_{4,3}=E & {\left[\int _ { G } \int _ { t } ^ { T } \left(\int _ { t } ^ { s } \left\{\frac{\partial}{\partial \gamma^{\prime}} \nabla\left(\widehat{H}_{0}(s, x)\right) \frac{\partial}{\partial \gamma^{\prime}} b(v, x)+D_{v}\left(\nabla\left(\frac{\partial}{\partial \gamma^{\prime}} \widehat{H}_{0}(s, x)\right)\right) \frac{\partial}{\partial \gamma^{\prime}} \sigma(v, x)\right.\right.\right.} \\
& \left.\left.\left.+\int_{\mathbb{R}_{0}} D_{v, z}\left(\nabla\left(\frac{\partial}{\partial \gamma^{\prime}} \widehat{H}_{0}(s, x)\right)\right) \frac{\partial}{\partial \gamma^{\prime}} \theta(v, x, z) \nu(d z)\right\} \nabla \widehat{Y}^{\beta}(v, x) d v\right) d s d x\right] ;
\end{aligned}
$$

This give since $\widehat{Y}^{\beta_{\alpha}}(t)=0$,

$$
\begin{aligned}
\frac{d}{d t} A_{1,1}= & -E\left[\int _ { G } \int _ { t } ^ { T } \left(\left\{\frac{\partial}{\partial \gamma} \widehat{H}_{0}(s, x) \frac{\partial}{\partial \gamma} b(t, x)+D_{t}\left(\frac{\partial}{\partial \gamma} \widehat{H}_{0}(s, x)\right) \frac{\partial}{\partial \gamma} \sigma(t, x)\right.\right.\right. \\
& \left.+\int_{\mathbb{R}} D_{t, z}\left(\frac{\partial}{\partial \gamma} \widehat{H}_{0}(s, x)\right) \frac{\partial}{\partial \gamma} \theta(t, x, z) \nu(d z)\right\} \widehat{Y}^{\beta_{\alpha}}(t, x) \\
& \left.\left.+\frac{\partial}{\partial \gamma} \widehat{H}_{0}(s, x) L \widehat{Y}^{\beta_{\alpha}}(t, x)\right) d s d x\right] ; \\
\frac{d}{d t} A_{1,2}=- & E\left[\int _ { G } \int _ { t } ^ { T } \left\{\frac{\partial}{\partial \gamma} \widehat{H}_{0}(s, x) \frac{\partial}{\partial u} b(t, x)+D_{t}\left(\frac{\partial}{\partial \gamma} \widehat{H}_{0}(s, x)\right) \frac{\partial}{\partial u} \sigma(t, x)\right.\right. \\
& \left.\left.+\int_{\mathbb{R}} D_{t, z}\left(\frac{\partial}{\partial \gamma} \widehat{H}_{0}(s, x)\right) \frac{\partial}{\partial u} \theta(t, x, z) \nu(d z)\right\} \alpha d t d x\right] \\
= & -E\left[\int _ { G } \frac { \partial } { \partial u } \left(\int_{t}^{T} \frac{\partial}{\partial \gamma} \widehat{H}_{0}(s, x) d s b(t, x, \widehat{u})+D_{t}\left(\int_{t}^{T} \frac{\partial}{\partial \gamma} \widehat{H}_{0}(s, x) d s\right) \sigma(t, x, \widehat{u})\right.\right. \\
& \left.\left.+\int_{\mathbb{R}} D_{t, z}\left(\int_{t}^{T} \frac{\partial}{\partial \gamma} \widehat{H}_{0}(s, x) d s\right) \theta(t, x, \widehat{u}) \nu(d z)\right) \alpha d x\right] \\
= & -E\left[\int_{G} \frac{\partial}{\partial u}\left(\widehat{H}_{1}(t, x, \widehat{u})\right) \alpha d x\right]
\end{aligned}
$$

where

$$
\begin{aligned}
\widehat{H}_{1}\left(s, x, \gamma, \gamma^{\prime}, u\right)= & \widehat{K}_{1}(s, x) b\left(s, x, \gamma, \gamma^{\prime}, u\right)+D_{s} \widehat{K}_{1}(s, x) \sigma\left(s, x, \gamma, \gamma^{\prime}, u\right) \\
& +\int_{\mathbb{R}} D_{s, z} \widehat{K}_{1}(s, x) \theta\left(s, x, \gamma, \gamma^{\prime}, z, u\right) \nu(d z)
\end{aligned}
$$


with

$$
\begin{aligned}
& \widehat{K}_{1}(t, x)=\int_{t}^{T} \frac{\partial}{\partial \gamma} \widehat{H}_{0}(s, x, \widehat{u}) d s \\
& \frac{d}{d t} A_{1,3}=-E\left[\int _ { G } \int _ { t } ^ { T } \left\{\frac{\partial}{\partial \gamma} \widehat{H}_{0}(s, x) \frac{\partial}{\partial \gamma^{\prime}} b(t, x)+D_{t}\left(\frac{\partial}{\partial \gamma} \widehat{H}_{0}(s, x)\right) \frac{\partial}{\partial \gamma^{\prime}} \sigma(t, x)\right.\right. \\
&+\left.\left.\int_{\mathbb{R}} D_{t, z}\left(\frac{\partial}{\partial \gamma} \widehat{H}_{0}(s, x)\right) \frac{\partial}{\partial \gamma^{\prime}} \theta(t, x, z) \nu(d z)\right\} \nabla \widehat{Y}^{\beta_{\alpha}}(t, x) d s d x\right] \\
& \frac{d}{d t} A_{2}(t)=-E\left[\int_{G} \frac{\partial}{\partial u}\left(\widehat{H}_{0}(t, x, \widehat{u})+f(t, x, \widehat{u})\right) \alpha d x\right] \\
& \frac{d}{d t} A_{3,1}=-E\left[\int _ { G } \int _ { t } ^ { T } \left(\left\{L^{*} \widehat{K}(s, x) \frac{\partial}{\partial \gamma} b(t, x)+D_{t}\left(L^{*} \widehat{K}(s, x)\right) \frac{\partial}{\partial \gamma} \sigma(t, x)\right.\right.\right. \\
&\left.+\int_{\mathbb{R}} D_{t, z}\left(L^{*} \widehat{K}(s, x)\right) \frac{\partial}{\partial \gamma} \theta(t, x, z) \nu(d z)\right\} \widehat{Y}^{\beta_{\alpha}}(t, x) \\
&\left.\left.+L^{*} \widehat{K}(s, x) L \widehat{Y}^{\beta_{\alpha}}(t, x)\right) d s d x\right] \\
& \frac{d}{d t} A_{3,2}=-E\left[\int_{G} \frac{\partial}{\partial u}\left(\widehat{H}_{2}(t, x, \widehat{u})\right) \alpha d x\right]
\end{aligned}
$$

where $L^{*}$ is the adjoint operator of $L$ and

$$
\begin{aligned}
\widehat{H}_{2}\left(s, x, \gamma, \gamma^{\prime}, u\right)= & \widehat{K}_{2}(s, x) b\left(s, x, \gamma, \gamma^{\prime}, u\right)+D_{s} \widehat{K}_{2}(s, x) \sigma\left(s, x, \gamma, \gamma^{\prime}, u\right) \\
& +\int_{\mathbb{R}} D_{s, z} \widehat{K}_{2}(s, x) \theta\left(s, x, \gamma, \gamma^{\prime}, z, u\right) \nu(d z)
\end{aligned}
$$

with

$$
\begin{aligned}
& \widehat{K}_{2}(t, x)=\int_{t}^{T} L^{*} \widehat{K}(s, x) d s \\
& \frac{d}{d t} A_{3,3}=-E\left[\int _ { G } \int _ { t } ^ { T } \left(\left\{L^{*} \widehat{K}(s, x) \frac{\partial}{\partial \gamma^{\prime}} b(t, x)+D_{t}\left(L^{*} \widehat{K}(s, x)\right) \frac{\partial}{\partial \gamma^{\prime}} \sigma(t, x)\right.\right.\right. \\
& \left.\left.\left.+\int_{\mathbb{R}} D_{t, z}\left(L^{*} \widehat{K}(s, x)\right) \frac{\partial}{\partial \gamma^{\prime}} \theta(t, x, z) \nu(d z)\right\} \nabla \widehat{Y}^{\beta_{\alpha}}(t, x)\right) d s d x\right] \\
& \frac{d}{d t} A_{4,1}=-E\left[\int _ { G } \int _ { t } ^ { T } \left(\left\{\nabla\left(\frac{\partial}{\partial \gamma^{\prime}} \widehat{H}_{0}(s, x)\right) \frac{\partial}{\partial \gamma} b(t, x)+D_{t}\left(\nabla\left(\frac{\partial}{\partial \gamma^{\prime}} \widehat{H}_{0}(s, x)\right)\right) \frac{\partial}{\partial \gamma} \sigma(t, x)\right.\right.\right. \\
& \left.+\int_{\mathbb{R}_{0}} D_{t, z}\left(\nabla\left(\frac{\partial}{\partial \gamma} \widehat{H}_{0}(s, x)\right)\right) \frac{\partial}{\partial \gamma} \theta(t, x, z) \nu(d z)\right\} \widehat{Y}^{\beta_{\alpha}}(t, x) \\
& \left.\left.+\nabla\left(\frac{\partial}{\partial \gamma^{\prime}} \widehat{H}_{0}(s, x)\right) L \widehat{Y}^{\beta_{\alpha}}(t, x)\right) d s d x\right] \\
& \frac{d}{d t} A_{4,2}=-E\left[\int_{G} \frac{\partial}{\partial u}\left(\widehat{H}_{3}(t, x, \widehat{u})\right) \alpha d x\right]
\end{aligned}
$$


where

$$
\begin{aligned}
\widehat{H}_{3}\left(s, x, \gamma, \gamma^{\prime}, u\right)= & \widehat{K}_{3}(s, x) b\left(s, x, \gamma, \gamma^{\prime}, u\right)+D_{s} \widehat{K}_{3}(s, x) \sigma\left(s, x, \gamma, \gamma^{\prime}, u\right) \\
& +\int_{\mathbb{R}} D_{s, z} \widehat{K}_{3}(s, x) \theta\left(s, x, \gamma, \gamma^{\prime}, z, u\right) \nu(d z)
\end{aligned}
$$

with

$$
\begin{gathered}
\widehat{K}_{3}(t, x)=\int_{t}^{T} \nabla\left(\frac{\partial}{\partial u} \widehat{H}_{0}(s, x, \widehat{u})\right) d s \\
\frac{d}{d t} A_{4,3}=-E\left[\int _ { G } \int _ { t } ^ { T } \left\{\nabla\left(\frac{\partial}{\partial \gamma^{\prime}} \widehat{H}_{0}(s, x)\right) \frac{\partial}{\partial \gamma^{\prime}} b(t, x)+D_{t}\left(\nabla\left(\frac{\partial}{\partial \gamma^{\prime}} \widehat{H}_{0}(s, x)\right)\right) \frac{\partial}{\partial \gamma^{\prime}} \sigma(t, x)\right.\right. \\
\left.\left.+\int_{\mathbb{R}_{0}} D_{t, z}\left(\nabla\left(\frac{\partial}{\partial \gamma^{\prime}} \widehat{H}_{0}(s, x)\right)\right) \frac{\partial}{\partial \gamma^{\prime}} \theta(t, x, z) \nu(d z)\right\} \nabla \widehat{Y}^{\beta_{\alpha}}(t, x) d s d x\right] ;
\end{gathered}
$$

Then we get by combining (28),(31), (33) and (26) with (15), we get that:

$$
E\left[\int_{G} \frac{\partial}{\partial u}\left\{\widehat{H}_{0}(s, x, \widehat{u})+\widehat{H}_{1}(s, x, \widehat{u})+\widehat{H}_{2}(s, x, \widehat{u})+\widehat{H}_{3}(s, x, \widehat{u})+f(s, x, \widehat{u})\right\} d x\right]=0
$$

Hence by the definition of $\widehat{p}(t, x)$, we have

$$
\widehat{p}(t, x)=\widehat{K}(t, x)+\widehat{K}_{1}(t, x)+\widehat{K}_{2}(t, x)+\widehat{K}_{3}(t, x)
$$

Then substitution of $\widehat{H}_{0}, \widehat{H}_{1}, \widehat{H}_{2}$ and $\widehat{H}_{3}$ in (39) gives

$$
\begin{aligned}
& E\left[\int _ { G } \frac { \partial } { \partial u } \left\{f(t, x, \Gamma, \widehat{u}, \omega)+p(t, x) b\left(t, x, \Gamma, \Gamma^{\prime}, \widehat{u}, \omega\right)+D_{t} p(t, x) \sigma\left(t, x, \Gamma, \Gamma^{\prime}, \widehat{u}, \omega\right)\right.\right. \\
& \left.\left.+\int_{\mathbb{R}} D_{t, z} p(t, x) \theta\left(t, x, \Gamma, \Gamma^{\prime}, \widehat{u}, z, \omega\right) \nu(d z)\right\} \alpha d x\right] \\
= & 0
\end{aligned}
$$

Since this holds for all bounded $\mathcal{E}_{t}$-measurable random variable $\alpha$, we conclude that

$$
E\left[\int_{G} \frac{\partial}{\partial u} \widehat{H}(t, x, \widehat{\Gamma}(t, x), \nabla \widehat{\Gamma}(t, x), \widehat{u}(t, x)) d x \mid \mathcal{E}_{t}\right]=0 \text { a.e. in }(t, x, \omega),
$$

which completes the proof.

\section{Applications}

In this Section we take aim at two applications of Theorem 3: The first one pertains to partial information optimal harvesting, whereas the other one refers to portfolio optimization under partial observation. 


\subsection{Partial information optimal harvesting}

Assume that $\Gamma(t, x)$ describes the density of a population (e.g. fish) at time $t \in(0, T)$ and at the location $x \in G \subset \mathbb{R}^{d}$. Further suppose that $\Gamma(t, x)$ is modelled by the stochastic-reaction diffusion equation

$$
\begin{aligned}
d \Gamma(t, x)= & {\left[\frac{1}{2} \Delta \Gamma(t, x)+b(t, \omega) \Gamma(t, x)-c(t)\right] d t+\sigma(t, \omega) \Gamma(t, x) d B_{t} } \\
& +\int_{\mathbb{R}} \theta(t, z, \omega) \Gamma(t, x) \widetilde{N}(d z, d t), \quad(t, x) \in[0, T] \times G \\
& \text { where } \Delta=\sum_{i=1}^{n} \frac{\partial^{2}}{\partial X_{i}^{2}} \text { is the Laplacian }
\end{aligned}
$$

with boundary condition

$$
\begin{aligned}
\Gamma(0, x) & =\xi(x), x \in \bar{G} \\
\Gamma(t, x) & =\eta(t, x),(t, x) \in(0, T) \times \partial G .
\end{aligned}
$$

where $b, \sigma, \theta$ are given $\mathcal{F}_{t}$-predictable processes. The process $c(t) \geq 0$ is our harvesting rate, which is assumed to be a $\mathcal{E}_{t}$-predictable admissible control.

We persue to maximize both expected cumulative utility of consumption and the terminal size of the population subject to the performance functional

$$
J(c)=E\left[\int_{G} \int_{0}^{T} \zeta(s) U(c(s)) d s d x+\int_{G} \xi \Gamma^{(c)}(T, x) d x\right]
$$

where $U:[0,+\infty] \rightarrow \mathbb{R}$ is a $C^{1}$ utility function, $\zeta(s)=\zeta(s, x, \omega)$ is an $\mathcal{F}_{t}$-predictable process and $\xi=\xi(\omega)$ is an $\mathcal{F}_{T}$-mesurable random variable.

We want to find an admissible control $\hat{c} \in A_{\mathcal{E}}$ such that

$$
\sup _{c \in A_{\mathcal{E}}} J(c)=J(\hat{c})
$$

Using the previous notation, we note that in this case, with $u=c$,

$$
f(t, x, \Gamma(t, x), c(t), \omega)=\zeta(s, \omega) U(c(t)) ; g(x, \Gamma(t, x), \omega)=\xi(\omega) \Gamma^{(c)}(t, x)
$$

Hence

$$
\begin{aligned}
K(t, x):= & \frac{\partial}{\partial \gamma} g(x, \Gamma(T, x), \omega)+\int_{t}^{T} \frac{\partial}{\partial \gamma} f(s, x, \Gamma(s, x), u(s, x), \omega) d s=\xi(\omega) \\
D_{t} K(t, x) & =D_{t, z} K(t, x)=0 \\
H_{0}(t, x, \gamma, c) & =\xi(\omega)(b(t, x, \omega) \gamma-c) \\
K_{1}(t, x) & =\int_{t}^{T} b(t, x, \omega) \xi(\omega) d t \\
K_{2}(t, x) & =K_{3}(t, x)=0
\end{aligned}
$$


Then

$$
p(t, x)=\xi(\omega)+\int_{t}^{T} b(t, x, \omega) \xi(\omega) d t
$$

and the Hamiltonian becomes

$$
\begin{aligned}
H(t, x, \gamma, c)= & \zeta(t) U(c)+p(t, x)(b(t, x) \Gamma(t, x)-c(t))+D_{t} p(t, x) \sigma(t) \\
& +\int_{\mathbb{R}_{0}} D_{t, z} p(t, x) \theta(t, z) \nu(d z)
\end{aligned}
$$

Then, $\hat{c} \in \mathcal{A}_{\mathcal{E}}$ is an optimal control for the problem (42) if we have:

$$
\begin{aligned}
0 & =E\left[\int_{G} \frac{\partial}{\partial c} H(t, x, \hat{\Gamma}(t, x), \hat{c}(t)) d x \mid \mathcal{E}_{t}\right] \\
& =E\left[\int_{G}\left\{\zeta(t) U^{\prime}(\hat{c}(t))-p(t, x)\right\} d x \mid \mathcal{E}_{t}\right] \\
& =U^{\prime}(\hat{c}(t)) E\left[\int_{G} \zeta(t, x) d x \mid \mathcal{E}_{t}\right]-E\left[\int_{G} p(t, x) d x \mid \mathcal{E}_{t}\right]
\end{aligned}
$$

We have proved a similar version of Theorem 4.2 in [14]:

Theorem 5 If there exists an optimal harvesting rate $\hat{c}(t)$ of problem (42), then it satisfies the equation

$$
U^{\prime}(\hat{c}(t)) E\left[\int_{G} \zeta(t, x) d x \mid \mathcal{E}_{t}\right]=E\left[\int_{G} p(t, x) d x \mid \mathcal{E}_{t}\right]
$$

\subsection{Application to optimal stochastic control of jump diffusion with partial observation}

In this Subsection we want to apply ideas of non-linear filtering theory in connection with Theorem 3 to solve a portfolio optimization problem, where the trader has limited access to market information (Example 6). As for general backround information on non-linear filtering theory the reader may e.g. consult [2]. For the concrete setting that follows below see also [10] and [11].

Suppose that the state process $X(t)=X^{(u)}(t)$ and the observation process $Z(t)$ are described by the following system of SDE's:

$$
\begin{gathered}
d X(t)=\alpha(X(t), u(t)) d t+\beta(X(t), u(t)) d B^{X}(t) \\
d Z(t)=h(t, X(t)) d t+d B^{Z}(t)+\int_{\mathbb{R}_{0}} \xi N_{\lambda}(d t, d \xi)
\end{gathered}
$$

where $\left(B^{X}(t) ; B^{Z}(t)\right) \in \mathbb{R}^{2}$ is a Wiener process independent of the initial value $X(0)$, and $N_{\lambda}$ is an integer valued random measure with predictable compensator

$$
\mu(d t, d \xi, \omega)=\lambda\left(t, X_{t}, \xi\right) d t \nu(d \varsigma)
$$

for a Lévy measure $\nu$ and an intensity rate function $\lambda(t, x, \varsigma)$, such that the increments of $N_{\lambda}$ are conditionally independent with respect to the filtration generated by $B_{t}^{X}$. Further $u(t)$ is 
a control process which takes values in a closed convex set $U \subset \mathbb{R}$ and which is adapted to a subfiltration $\mathcal{E}_{t} \subset \mathcal{G}_{t}$. Here $\mathcal{G}_{t}$ is generated by the observation process $Z(t)$. The coefficients $\alpha: \mathbb{R} \times U \longrightarrow \mathbb{R}, \beta: \mathbb{R} \times U \longrightarrow \mathbb{R}, \lambda: \mathbb{R}_{+} \times \mathbb{R} \times \mathbb{R}_{0} \longrightarrow \mathbb{R}$ and $h: \mathbb{R}_{+} \times \mathbb{R} \longrightarrow \mathbb{R}$ are twice continuously differentiable.

In what follows we shall assume that a strong solution $X_{t}=X_{t}^{(u)}$ of (46), if it exists, takes values in a given Borel set $G \subseteq \mathbb{R}$. Let us introduce the performance functional

$$
J(u):=E\left[\int_{0}^{T} f(X(t), Z(t), u(t)) d t+g(X(T), Z(T))\right],
$$

where $f: G \times \mathbb{R} \times U \longrightarrow \mathbb{R}, g: G \times \mathbb{R} \longrightarrow \mathbb{R}$ are lower bounded $C^{1}$ functions. We want to find the maximizer $u^{*}$ of $J$, that is

$$
J^{*}=\sup _{u \in \mathcal{A}} J(u)=J\left(u^{*}\right)
$$

where $\mathcal{A}$ is the set of admissible controls consisting of $\mathcal{E}_{t}$-predictable controls $u$ such that (46) admits a unique strong solution.

We shall now briefly outline how the optimal control problem (47) for SDE's with partial observation can be transformed into one for SPDE's with complete information. See e.g. [2] and [10] for details. In the sequel we assume that $\lambda(t, x, \xi)>0$ for all $t, x, \xi$ and that the exponential process

$$
\begin{aligned}
M_{t}:= & \exp \left\{\int_{0}^{t} h(X(s)) d B^{Z}(s)-\frac{1}{2} \int_{0}^{t} h^{2}(X(s)) d s\right. \\
& \left.+\int_{0}^{t} \int_{\mathbb{R}_{0}} \log \lambda(s, X(s), \xi) N_{\lambda}(d s, d \xi)+\int_{0}^{t} \int_{\mathbb{R}_{0}}[1-\lambda(s, X(s), \xi)] d s \nu(d \xi)\right\} ; t \geq 0
\end{aligned}
$$

is well defined and a martingale. Define the change of measure

$$
d Q=M_{T} d P
$$

and set

$$
N_{T}=M_{T}^{-1}
$$

Using the Girsanov theorem for random measures and the uniqueness of semimartingale characteristics (see, e.g. [8]), one sees that the processes (46) get decoupled under the measure $Q$ in the sense that system (46) transforms to

$$
\begin{gathered}
d X(t)=\alpha(X(t), u(t)) d t+\beta(X(t), u(t)) d B^{X}(t) \\
d Z(t)=d B(t)+d L(t)
\end{gathered}
$$

where $Z(t)$ is a Levy process independent of Brownian motion $B^{X}(t)$, and consequently independent of $X(t)$, under $Q$. Here

$$
B(t)=B^{Z}(t)-\int_{0}^{t} h(X(s)) d s
$$


is the Brownian motion part and

$$
L(t)=\int_{0}^{t} \int_{\mathbb{R}_{0}} \xi N(d t, d \xi)
$$

is the pure jump component associated to the Poisson random measure $N(d t, d \xi)=N_{\lambda}(d t, d \xi)$ with compensator given by $d s \nu(d \xi)$.

Define the differential operator $A=A_{z, u}$ by

$$
A \phi(x)=A_{u} \phi(x)=\alpha(x, u) \frac{d \phi}{d x}(x)+\frac{1}{2} \beta^{2}(x, u) \frac{d^{2} \phi}{d x^{2}}(x)
$$

for $\phi \in C_{0}^{2}(\mathbb{R})$. Hence $A_{u}$ is the generator of $X(t)$, if $u$ is constant. Set

$$
a(x, u)=\frac{1}{2} \beta^{2}(x, u) .
$$

Then the adjoint operator $A^{*}$ of $A$ is given by

$$
A^{*} \phi=\frac{\partial}{\partial x}\left(a(x, u) \frac{d \phi}{d x}(x)\right)+\frac{\partial}{\partial x}\left(\frac{\partial a}{\partial x}(x, u) \phi(x)\right)-\frac{\partial}{\partial x}(a(x, u) \phi(x)) .
$$

Let us assume that the initial condition $X(0)$ has a density $p_{0}$ and that there exists a unique strong solution $\Phi(t, x)$ of the following SPDE (Zakai equation)

$$
d \Phi(t, x)=A^{*} \Phi(t, x) d t+h(x) \Phi(t, x) d B(t)+\int_{\mathbb{R}_{0}}[\lambda(t, x, \xi)-1] \Phi(t, x) \widetilde{N}(d t, d \xi)
$$

with

$$
\Phi(0, x)=p_{0}(x)
$$

Then $\Phi(t, x)$ is the unnormalized conditional density of $X(t)$ given $\mathcal{G}_{t}$ and satisfies:

$$
E_{Q}\left[\phi(X(t)) N_{t} \mid \mathcal{G}_{t}\right]=\int_{\mathbb{R}} \phi(x) \Phi(t, x) d x
$$

for all $\phi \in C_{b}(\mathbb{R})$.

Using (51) and (50) under the change of measure $Q$ and the definition of the performance functional we obtain that

$$
\begin{aligned}
J(u) & =E\left[\int_{0}^{T} f(X(t), Z(t), u(t)) d t+g(X(T), Z(T))\right] \\
& =E_{Q}\left[\left\{\int_{0}^{T} f(X(t), Z(t), u(t)) d t+g(X(T), Z(T))\right\} N_{T}\right] \\
& =E_{Q}\left[\int_{0}^{T} f(X(t), Z(t), u(t)) N_{t} d t+g(X(T), Z(T)) N_{T}\right] \\
& =E_{Q}\left[\int_{0}^{T} E_{Q}\left[f(X(t), Z(t), u(t)) N_{t} \mid \mathcal{G}_{t}\right] d t+E_{Q}\left[g(X(T), Z(T)) N_{T} \mid \mathcal{G}_{t}\right]\right] \\
& =E_{Q}\left[\int_{0}^{T} \int_{G} f(x, Z(t), u(t)) \Phi(t, x) d x d t+\int_{G} g(x, Z(T)) \Phi(T, x) d x\right]
\end{aligned}
$$


The observation process $Z(t)$ is a $Q$-Lévy process. Hence the partial observation control problem (47) reduces to a SPDE control problem under complete information. More precisely, our control problem is equivalent to the maximization problem

$$
\sup _{u} E_{Q}\left[\int_{0}^{T} \int_{G} f(x, Z(t), u(t)) \Phi(t, x) d x d t+\int_{G} g(x, Z(T)) \Phi(T, x) d x\right]
$$

where $\Phi$ solves the SPDE (50). So the latter problem can be tackled by means of the maximum principle of Section 2.

For convenience, let us impose that $a$ in (48) is independent of the control, i.e.

$$
a(x, u)=a(x) .
$$

Denote by $\mathcal{A}_{1}$ the set $u \in \mathcal{A}$ for which (50) has a unique solution. Consider the general stochastic Hamiltonian (if existent) of the control problem (52) given by

$$
\begin{aligned}
H\left(t, x, \phi, \phi^{\prime}, u, \omega\right)= & f(t, x, Z(t), u) \phi+p(t, x) b\left(t, x, \phi, \phi^{\prime}, u\right)+D_{t} p(t, x) h(x) \phi \\
& +\int_{\mathbb{R}_{0}} D_{t, z} p(t, x)[\lambda(t, x, \xi)-1] \phi \nu(d z),
\end{aligned}
$$

where

$$
b\left(t, x, \phi, \phi^{\prime}, u\right)=\left(\frac{d^{2} a}{d x^{2}}(x)-\alpha(x, u)\right) \phi+\left(\frac{d a}{d x}(x)-\alpha(x, u)\right) \phi^{\prime}
$$

and where $p(t, x)$ is defined as in (13) with

$$
g(x, \phi, \omega)=g(x, Z(T)) \phi
$$

and

$$
L \psi(x)=a(x) \frac{d^{2} \psi}{d x^{2}}(x), \psi \in C_{0}^{2}(\mathbb{R})
$$

Assume that the conditions (i)-(v) of Section 3.2 hold with respect to (52) for controls $u \in \mathcal{A}_{1}$. Then by the general stochastic maximum principle (Theorem 3 ) applied to the partial information control problem (47) we find that

$$
E\left[\int_{G} \frac{\partial}{\partial u} \widehat{H}\left(t, x, \widehat{\Phi}, \widehat{\Phi}^{\prime}, \hat{u}, \omega\right) d x \mid \mathcal{E}_{t}\right]=0
$$

if $\hat{u} \in \mathcal{A}_{1}$ is an optimal control

Example 6 (Optimal consumption with partial observation) Let us illustrate the maximum principle by inquiring into the following portfolio optimization problem with partial observation: Assume the wealth $X(t)$ at time $t$ of an investor is modelled by

$$
d X(t)=[\mu X(t)-u(t)] d t+\sigma X(t) d B^{X}(t), 0 \leq t \leq T
$$


where $m \in \mathbb{R}, \sigma \neq 0$ are constants, $B^{X}(t)$ a Brownian motion and $u(t) \geq 0$ the consumption rate. Suppose that the initial value $X(0)$ has the density $p_{0}(x)$ and that $u(t)$ is adapted to the filtration $\mathcal{G}_{t}$ generated by the observation process

$$
d Z(t)=m X(t) d t+d B^{Z}(t)+\int_{\mathbb{R}_{0}} \xi N_{\lambda}(d t, d \xi), Z(0)=0
$$

where $m$ is a constant. As before we require that $\left(B^{X}(t), B^{Z}(t)\right)$ is a Brownian motion independent of the initial value $X(0)$, and that $N_{\lambda}$ is an integer valued random measure as described in (??). Further, let us restrict the wealth process $X(t)$ to be bounded from below by a treshold $\zeta>0$ for $0 \leq t \leq T$. The investor intends to maximize the expected utility of his consumption and terminal wealth according to the peformance criterion

$$
J(u)=E\left[\int_{0}^{T} \frac{u^{r}(t)}{r} d t+\theta X^{r}(T)\right], r \in(0,1), \quad \theta>0
$$

So we are dealing with a partial observation control problem of the type $(47)($ for $G=[\zeta, \infty))$. Here, the operator $A$ in (50) has the form

$$
A \phi(x)=\frac{1}{2} \sigma^{2} x^{2} \phi^{\prime \prime}(x)+[\mu x-u] \phi^{\prime}(x)
$$

(where 1 denotes the differentiation which respect to $x$ ) and hence

$$
A^{*} \phi(x)=\frac{1}{2} \sigma^{2} x^{2} \phi^{\prime \prime}(x)-[\mu x-u] \phi^{\prime}(x)-\mu \phi(x) .
$$

Therefore the Zakai equation becomes

$$
\begin{aligned}
d \Phi(t, x)= & {\left[\frac{1}{2} \sigma^{2} x^{2} \Phi^{\prime \prime}(t, x)-[\mu x-u] \Phi^{\prime}(t, x)-\mu \Phi(t, x)\right] d t+x \Phi(t, x) d B(t) } \\
& +\int_{\mathbb{R}_{0}}[\lambda(t, x, \xi)-1] \Phi(t, x) \widetilde{N}(d t, d \xi) \\
\Phi(t, x)= & p_{0}(x), x>\zeta \\
\Phi(t, 0)= & 0 ; \quad t \in(0, T)
\end{aligned}
$$

where $\widetilde{N}(d t, d \xi)$ is a compensated Poisson random measure under the corresponding measure $Q$. Since $L \psi=\frac{1}{2} \sigma^{2} x^{2} \frac{d^{2} \psi}{d x^{2}}(x)$ is uniformly elliptic for $x>\zeta$ there exists a unique strong solution of (58). Further one verifies that condition (iv) of Section 3.2 is fulfilled. See [2]. So our problem amounts to finding an admissible $\hat{u} \in \mathcal{A}_{1}$ such that

$$
J_{1}(\hat{u})=\sup _{u \in \mathcal{A}_{1}} J_{1}(u)
$$

where

$$
J_{1}(u)=E_{Q}\left[\int_{0}^{T} \int_{G} \frac{u^{r}(t)}{r} \Phi(t, x) d x d t+\int_{V} \theta x^{r} \Phi(T, x) d x\right]
$$


Using the notation of (53) we see that

$$
\begin{aligned}
& f(x, Z(t), u(t))=\frac{u^{r}(t)}{r} \\
& g(x, Z(T))=\theta x^{r} \\
& L^{*} \Phi(t, x)=\frac{1}{2} \sigma^{2} x^{2} \frac{\partial^{2}}{\partial x^{2}} \Phi(t, x) \\
& K(t, x)=\theta x^{r}(T)+\int_{t}^{T} \frac{u^{r}(t)}{r} d s \\
& H_{0}\left(t, x, \phi, \phi^{\prime}, u\right)=\left[(-\mu x-u) \phi^{\prime}(t, x)-\mu \phi(t, x)\right] K(t, x)+D_{t} K(t, x) x \phi \\
& +\int_{\mathbb{R}_{0}} D_{t, z} K(t, x)[\lambda(t, x, \xi)-1] \phi \nu(d \xi) \\
& K_{1}(t, x)=\int_{t}^{T}\left\{-\mu K(s, x)+D_{s} K(s, x) x+\int_{\mathbb{R}_{0}} D_{s, z} K_{1}(s, x)[\lambda(s, x, \xi)-1] \nu(d \xi)\right\} d s \\
& K_{2}(t, x)=\int_{t}^{T} \frac{1}{2} \sigma^{2} x^{2} \frac{\partial^{2}}{\partial x^{2}} K(s, x) d s \\
& K_{3}(t, x)=\int_{t}^{T} \frac{\partial}{\partial x}[(-\mu x-u) K(s, x)] d s \\
& p(t, x)=K(t, x)+\int_{t}^{T}\left\{\frac{1}{2} \sigma^{2} x^{2} \frac{\partial^{2}}{\partial x^{2}} K(s, x)+\frac{\partial}{\partial x}[(-\mu x-u) K(s, x)]\right. \\
& \left.-\mu K(s, x)+D_{s} K(s, x) x+\int_{\mathbb{R}_{0}} D_{s, z} K_{1}(s, x)[\lambda(s, x, \xi)-1] \nu(d \xi)\right\} d s .
\end{aligned}
$$

So the Hamiltonian is well-defined and becomes

$$
\begin{aligned}
H\left(t, x, \phi, \phi^{\prime}, u\right)= & \frac{u^{r}(t)}{r} \phi+\left[(-\mu x-u) \phi^{\prime}(t, x)-\mu \phi(t, x)\right] p(t, x) \\
& +D_{t} p(t, x) x \phi+\int_{\mathbb{R}_{0}} D_{t, z} p(t, x)[\lambda(t, x, \xi)-1] \phi \nu(d \xi)
\end{aligned}
$$

Hence, if $\hat{u}$ is an optimal control of the problem (47), then it follows from (54) and (51) that

$$
\begin{aligned}
0 & =E_{Q}\left[\int_{G} \frac{\partial}{\partial u} H\left(t, x, \widehat{\Phi}, \widehat{\Phi}^{\prime}, \widehat{u}\right) d x \mid \mathcal{G}_{t}\right] \\
& =E_{Q}\left[\int_{G}\left\{u^{r-1}(t) \widehat{\Phi}(t, x)+\widehat{\Phi}^{\prime}(t, x) \widehat{p}(t, x)\right\} d x \mid \mathcal{G}_{t}\right]
\end{aligned}
$$

Thus we get

$$
u^{r-1}(t)=-\frac{E_{Q}\left[\int_{G} \widehat{\Phi}^{\prime}(t, x) \widehat{p}(t, x) d x \mid \mathcal{G}_{t}\right]}{E_{Q}\left[\int_{G} \widehat{\Phi}(t, x) d x \mid \mathcal{G}_{t}\right]}
$$


Using integration by parts and (51) implies that

$$
\begin{aligned}
u^{*}(t) & =\left(-\frac{E_{Q}\left[\int_{G} \widehat{\Phi}^{\prime}(t, x) \widehat{p}(t, x) d x \mid \mathcal{G}_{t}\right]}{E_{Q}\left[\int_{G} \widehat{\Phi}(t, x) d x \mid \mathcal{G}_{t}\right]}\right)^{\frac{1}{r-1}} \\
& =\left(\frac{E_{Q}\left[\int_{G} \widehat{\Phi}(t, x) \widehat{p}^{\prime}(t, x) d x \mid \mathcal{G}_{t}\right]}{E_{Q}\left[\int_{G} \widehat{\Phi}(t, x) d x \mid \mathcal{G}_{t}\right]}\right)^{\frac{1}{r-1}} \\
& =\left(\frac{E_{Q}\left[\widehat{p}^{\prime}(t, X(t)) N_{t} \mid \mathcal{G}_{t}\right]}{E_{Q}\left[N_{t} \mid \mathcal{G}_{t}\right]}\right)^{\frac{1}{r-1}} \\
& =E\left[\widehat{p}^{\prime}(t, X(t)) \mid \mathcal{G}_{t}\right]^{\frac{1}{r-1}}
\end{aligned}
$$

So if $u^{*}(t)$ maximizes (55) then $u^{*}(t)$ necessarily sastisfies

$$
u^{*}(t)=E\left[\widehat{p}^{\prime}(t, X(t)) \mid \mathcal{G}_{t}\right]^{\frac{1}{r-1}} .
$$

Remark 7 Note that the last example cannot be treated within the framework of [14], since the random measure $N_{\lambda}(d t, d \xi)$ is not necessarily a functional of a Lévy process. Let us also mention that the SPDE maximum principle studied in [13] does not apply to Example 6. This is due to the fact the corresponding Hamiltonian in [13] fails to be concave.

\section{References}

[1] Baghery, F., Øksendal, B.: A maximum principle for stochastic control with partial information. Stochastic Analysis and Applications 25, p. 705-717 (2007).

[2] Bensousssan, A.: Maximum principle and dynamic programming Approaches of the optimal control of partially observed diffusions. Stochastics 9 (1983).

[3] Carmona, R., Tehranchi, M.: Interest Rate Models: an Infinite Dimensional Stochastic Analysis Perspective. Springer (2006).

[4] Da Prato, G., Zabczyk, J.: Stochastic Equations in Infinite Dimensions. Cambridge University Press (1992).

[5] Di Nunno, G., Øksendal, B., Proske, F.: Malliavin Calculus for Lévy processes with Applications to Finance. Springer. To appear (2008).

[6] Di Nunno, G., Meyer-Brandis, T., Øksendal, B., Proske, F.: Malliavin calculus and anticipative Itô formulae for Lévy processes. Inf. dim. Anal. Quant. Probab. 8, p. 235258 (2005).

[7] Framstad, N., Øksendal, B., Sulem, A.: Stochastic maximum principle for optimal control of jump diffusions and applications to finance. J. Optimization Theory and Appl. 121 (1), p. 77-98 (2004). Errata: J. Optimization Theory and Appl. 124 (2), p. 511-512 (2005). 
[8] Jacod, J., Shiryaev, A.N.: Limit Theorems for Stochastic Processes. Springer (1987).

[9] Kallianpur, G., Xiong, J.: Stochastic Differential Equations in Infinite Dimensional Spaces. IMS Lecture Notes-Monograph Series, Vol. 26 (1995).

[10] Mandrekar, V., Meyer-Brandis T.; Proske F. (2007): A Bayes formula for non-linear filtering with Gaussian and Cox noise, Pue Mathematics Preprint series, University of Oslo.

[11] Meyer-Brandis, T., Proske, F.: Explicit solution of a non-linear filtering problem for Lévy processes with application to finance. Appl. Math. Optim. 50, p. 119-134 (2004).

[12] Nualart, D.: The Malliavin Calculus and Related Topics. Springer (1995).

[13] Øksendal, B., Proske, F., Zhang, T.: Backward stochastic partial differential equations with jumps and application to optimal control of random jump fields. Stochastics 77 (5), p. 381-399 (2005).

[14] Øksendal, B., Zhou, X.: A Malliavin calculus approach to a general maximum principle for stochastic control. Manuscript in preparation. University of Oslo (2007).

[15] Sanz-Solé, M.: Malliavin Calculus with Applications to Stochastic Partial Differential Equations. CRC Press (2005).

[16] Tang, S.: The maximum principle for partially observed optimal control of stochastic differential equations.. SIAM Journal on Control and Optimization 36 (5), p. 1596-1617 (1998).

[17] Zhou, X.: On the necessary conditions of optimal controls for stochastic partial differential equations. SIAM Journal on Control and Optimization 31, 86), p. 1462-1478 (1993). 